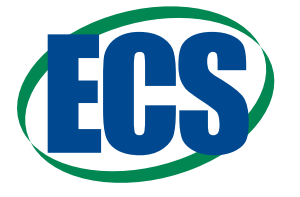

Focus Issue of Selected Papers from IMLB 2016 with Invited Papers Celebrating 25 Years of Lithium lon Batieries

\title{
Amorphous Lithium Lanthanum Titanate for Solid-State Microbatteries
}

\author{
Jungwoo Z. Lee, ${ }^{a, *}$ Ziying Wang, ${ }^{a}$ Huolin L. Xin, ${ }^{b}$ Thomas A. Wynn,,* \\ and Ying Shirley Meng ${ }^{\mathrm{a}, \mathrm{c}, * *, \mathrm{z}}$ \\ ${ }^{a}$ Department of NanoEngineering, University of California, San Diego, La Jolla, California 92093, USA \\ ${ }^{b}$ Center for Functional Nanomaterials, Brookhaven National Laboratory, Upton, New York 11973, USA \\ ${ }^{c}$ Materials Science and Engineering Program, University of California, San Diego, La Jolla, California 92093, USA
}

Lithium lanthanum titanate (LLTO) is a promising solid state electrolyte for solid state batteries due to its demonstrated high bulk ionic conductivity. However, crystalline LLTO has a relatively low grain boundary conductivity, limiting the overall material conductivity. In this work, we investigate amorphous LLTO (a-LLTO) thin films grown by pulsed laser deposition (PLD). By controlling the background pressure and temperature we are able to optimize the ionic conductivity to $3 \times 10^{-4} \mathrm{~S} / \mathrm{cm}$ and electronic conductivity to $5 \times 10^{-11} \mathrm{~S} / \mathrm{cm}$. XRD, TEM, and STEM/EELS analysis confirm that the films are amorphous and indicate that oxygen background gas is necessary during the PLD process to decrease the oxygen vacancy concentration, decreasing the electrical conductivity. Amorphous LLTO is deposited onto high voltage $\mathrm{LiNi}_{0.5} \mathrm{Mn}_{1.5} \mathrm{O}_{4}$ (LNMO) spinel cathode thin films and cycled up to $4.8 \mathrm{~V}$ vs. Li showing excellent capacity retention. These results demonstrate that a-LLTO has the potential to be integrated into high voltage thin film batteries.

(C) The Author(s) 2016. Published by ECS. This is an open access article distributed under the terms of the Creative Commons Attribution Non-Commercial No Derivatives 4.0 License (CC BY-NC-ND, http://creativecommons.org/licenses/by-nc-nd/4.0/), which permits non-commercial reuse, distribution, and reproduction in any medium, provided the original work is not changed in any way and is properly cited. For permission for commercial reuse, please email: oa@electrochem.org. [DOI: 10.1149/2.0411701jes] All rights reserved.

(cc) BY-NC-ND

Manuscript submitted September 9, 2016; revised manuscript received October 26, 2016. Published December 16, 2016. This was Paper 23 presented at the Chicago, Illinois, Meeting of the IMLB, June 19-24, 2016. This paper is part of the Focus Issue of Selected Papers from IMLB 2016 with Invited Papers Celebrating 25 Years of Lithium Ion Batteries.

Next generation lithium-ion batteries will require a broad range of energies to meet the challenges of portable electronic storage from electric vehicles to microelectromechanical systems (MEMS). The cost per Watt-hour of commercial batteries have shown incremental improvement due to better manufacturing design, but drastic increases in energy and power density are needed to satisfy projected demand. ${ }^{1}$ Solid-state electrolytes are researched heavily because they have the potential to improve capacity loss, cycle lifetime, operation temperature, and safety. Lithium Phosphorous Oxynitride (LiPON) based thin-film solid-state batteries have excellent cycle life and are currently commercialized. ${ }^{2,3}$ However, LiPON has a relatively low ionic conductivity $\left(1 \times 10^{-6} \mathrm{~S} / \mathrm{cm}\right)$ and other solid electrolytes have demonstrated conductivity several orders of magnitude higher. ${ }^{4,5}$

Lithium lanthanum titanate (LLTO) is a promising solid-state electrolyte due to its high bulk ionic conductivity $\left(\sim 10^{-3} \mathrm{~S} / \mathrm{cm}\right)$ at room temperature, negligible electronic conductivity, and high voltage, atmospheric, and temperature stabilities. ${ }^{6-8}$ Extensive fundamental studies have been carried out to demonstrate this high ionic conductivity, elucidate the crystal structure, and determine the mechanism of lithium ion conduction. ${ }^{9-12}$ However, there are fundamental impediments to the implementation of crystalline LLTO into an actual device. One key issue is that crystalline LLTO has a relatively low grain boundary ionic conductivity $\left(<10^{-5} \mathrm{~S} / \mathrm{cm}\right)$, lowering the effective material ionic conductivity. ${ }^{6}$ In addition, crystalline LLTO is unstable in contact with lithium metal because lithium will easily insert reducing $\mathrm{Ti}^{4+}$ to $\mathrm{Ti}^{3+}$, thus increasing electronic conductivity. ${ }^{13,14}$

Fortunately, amorphous LLTO has not only been shown to overcome these barriers, the lower energy constraints of fabricating amorphous LLTO opens up numerous thin film synthesis techniques. Amorphous LLTO thin films have been synthesized by pulsed laser deposition (PLD), RF magnetron sputtering, E-beam evaporation, atomic layer deposition, chemical solution deposition and sol-gel synthesis. ${ }^{15-24}$ Furusawa et al. demonstrated amorphous LLTO thin films deposited via PLD with higher ionic conductivity $\left(8.98 \times 10^{-4}\right.$
$\mathrm{S} / \mathrm{cm}$ ) than polycrystalline thin films. ${ }^{15}$ They suggest that this is likely due to the lack of grain boundaries and open disordered structure. However, these films also suffer from a high electronic conductivity of $4.0 \times 10^{-5} \mathrm{~S} / \mathrm{cm}$. Furthermore, Ahn and Yoon deposited amorphous LLTO thin films by PLD with lower ionic conductivity $\left(2.0 \times 10^{-5}\right.$ $\mathrm{S} / \mathrm{cm}$ ) and found that there was no electronic conductivity degradation when in contact with lithium metal. ${ }^{19}$ Zheng et al. also demonstrated that amorphous LLTO powders by sol-gel synthesis remain ionically conductive in contact with lithium metal even though it undergoes the same lithium insertion and $\mathrm{Ti}^{4+}$ to $\mathrm{Ti}^{3+}$ reduction. ${ }^{8}$ They hypothesize that this phenomenon is due to local atomic disorder in the amorphous case that localize electronic states.

Lastly, amorphous LLTO thin films have a large voltage stability window, which opens a pathway for high-voltage cathode materials, such as $\mathrm{LiNi}_{0.5} \mathrm{Mn}_{1.5} \mathrm{O}_{4}$ (LNMO) spinel. High-voltage cathodes have the potential to greatly improve the energy density of lithium ion batteries, but current liquid electrolytes face stability issues at high voltage due to strong oxidation reactions. ${ }^{25}$ With proper optimization, amorphous LLTO is a high ion conductive solid-state electrolyte with the potential to enable high voltage batteries with lithium metal anode.

Therefore, in this work, we investigate amorphous LLTO thin films grown by PLD for high voltage thin film batteries. By controlling the background pressure and temperature we are able to grow films with high ionic conductivity $\left(3 \times 10^{-4} \mathrm{~S} / \mathrm{cm}\right)$ several orders of magnitude higher than its electronic conductivity. Grazing incidence X-ray diffraction (GIXRD), transmission electron microscopy (TEM), and electron energy loss spectroscopy (EELS) analysis confirms that the films are amorphous and indicates that sufficient oxygen background gas is necessary during PLD to minimize oxygen vacancy concentration, which lowers the electrical conductivity. Amorphous LLTO is deposited onto high voltage $\mathrm{LiNi}_{0.5} \mathrm{Mn}_{1.5} \mathrm{O}_{4}$ (LNMO) spinel thin films and cycled up to $4.8 \mathrm{~V}$ vs. Li showing excellent capacity retention. These results demonstrate that a-LLTO is stable across the full voltage range and has minimal adverse interfacial reactions with LNMO.

\section{Experimental}

*Electrochemical Society Student Member

**Electrochemical Society Member

zE-mail: shmeng@ucsd.edu
LLTO synthesis. - The $\mathrm{Li}_{0.5} \mathrm{La}_{0.5} \mathrm{TiO}_{3}$ (LLTO) target was synthesized via solid state reaction consistent with previous reports. ${ }^{15,19,26}$ 
Stoichiometric amounts of $\mathrm{Li}_{2} \mathrm{CO}_{3}$ (Sigma Aldrich, 99.8\%), $\mathrm{La}_{2} \mathrm{O}_{3}$ (Sigma Aldrich, 99.9\%), and $\mathrm{TiO}_{2}$ (Fisher Scientific, 95.0\%) powders were ground with an agate mortar and pestle and calcined in an alumina crucible in a box furnace under ambient atmosphere. Samples were heated to $1200^{\circ} \mathrm{C}$, held for 6 hours, and then cooled back to room temperature at a ramp rate of $5^{\circ} \mathrm{C} / \mathrm{min}$. The powder was ground again and pressed in a 1-1/8 dye press with 10 tons of pressure for 5 minutes. The formed pellet was then sintered at $1300^{\circ} \mathrm{C}$ for 5 hours, using a ramp rate of $5^{\circ} \mathrm{C} / \mathrm{min}$.

$\mathrm{X}$-ray diffraction (XRD) analysis of the resulting pellet was collected using a Rigaku SmartLab X-ray diffractometer with $\mathrm{Cu} \mathrm{K} \alpha$ source operating at $30 \mathrm{kV}$ and $15 \mathrm{~mA}$ with a step size of $0.05^{\circ}$ at $1 \%$ min, scanning over $10-80^{\circ}$. Reitveld refinement was used to determine the crystalline phases. Both sides of the LLTO pellet were coated with $100 \mathrm{~nm}$ of Au using a Denton Discovery 18 Sputtering System and a Biologic SP-200 Potentiostat was used to conduct electrochemical impedance spectroscopy (EIS). The frequency range was $7 \mathrm{MHz}$ to $100 \mathrm{mHz}$ with an amplitude of $10 \mathrm{mV}$ and data was fit with a complex non-linear least square fitting method. The metal contacts were subsequently sanded off and the polished pellet was used for pulsed laser deposition (PLD).

Pulsed laser deposition of LLTO.-Thin films were grown using an Excel Instruments PLD-STD-12 chamber and $248 \mathrm{~nm} \mathrm{KrF}$ Lambda Physik LPX-Pro 210 excimer laser. Before deposition the chamber was pumped down to a baseline pressure of $<2.0 \times 10^{-6}$ Torr. Amorphous LLTO thin films were deposited at a range of pressures and temperatures, with a constant $\sim 2 \mathrm{~J} / \mathrm{cm}^{2}$ energy density and $4 \mathrm{~Hz}$ laser frequency. Amorphous LLTO was deposited on 2 different substrates for various analyses. For interdigitated samples, 2 electronically isolated interdigitated contact pads were sputtered on polished $\mathrm{SiO}_{2} / \mathrm{Si}$ similar to Furusawa et al. ${ }^{15}$ The interdigitated contact finger widths were $\sim 120 \mu \mathrm{m}$ with $\sim 80 \mu \mathrm{m}$ spacing and the films were $\sim 300 \mathrm{~nm}$ thick. Resulting measurements correspond to conduction parallel to the thin film surface. For vertical samples, $\sim 1.2 \mu \mathrm{m}$ of amorphous LLTO was deposited on Pt coated $\mathrm{SiO}_{2} / \mathrm{Si}$. Another layer of Pt was deposited via DC sputtering to fabricate Pt/a-LLTO/Pt symmetric cells in the architecture necessary to eventually fabricate a solid-state battery device.

A Biologic SP-200 Potentiostat was again used to measure the electronic conductivity by DC polarization and the ionic conductivity by electrochemical impedance spectroscopy (EIS). The frequency range was $3 \mathrm{MHz}$ to $100 \mathrm{mHz}$ with an amplitude of $10 \mathrm{mV}$ and data fitted with a complex non-linear least square fitting method. For low temperature EIS measurements the samples were placed in an Espec temperature chamber.

Electrochemical testing. - The $\mathrm{LiNi}_{0.5} \mathrm{Mn}_{1.5} \mathrm{O}_{4}$ (LNMO) target was synthesized by solid state reaction previously reported. ${ }^{27} \mathrm{MnO}_{2}$ (Sigma Aldrich, 99.99\%), $\mathrm{NiO}$ (Sigma Aldrich, 99.99\%), and $\mathrm{LiOH}$ (Sigma Aldrich, 98.0\%) powders were mixed, pressed in a 1-1/8 dye press with 10 tons of pressure for 10 minutes, and calcined in a box furnace under ambient atmosphere at $750^{\circ} \mathrm{C}$ for 24 hours with a ramp rate of $3^{\circ} \mathrm{C} / \mathrm{min}$. Afterwards, the powder was ball milled for 5 hours, pressed, and then sintered at $900^{\circ} \mathrm{C}$ for 2 hours using a ramp rate of $3^{\circ} \mathrm{C} / \mathrm{min}$. The resulting pellet was sanded and used as a target for pulsed laser deposition (PLD). The LNMO target had excess lithium $(1.3 \times \mathrm{Li})$ to compensate for $\mathrm{Li}$ loss during PLD. LNMO thin films were deposited on Pt-coated $\mathrm{Al}_{2} \mathrm{O}_{3}$ substrates at $600^{\circ} \mathrm{C}, 0.2$ Torr $\mathrm{O}_{2}$ partial pressure, $\sim 2 \mathrm{~J} / \mathrm{cm}^{2}$ energy density, and laser pulse frequency of $10 \mathrm{~Hz}$ for 40 minutes.

LNMO and LNMO/a-LLTO thin film electrodes were assembled into SS316L 2032 coin cells in a glove box purged with high purity argon $(99.9995 \%)$ and maintained with oxygen and water vapor levels at or less than $5 \mathrm{ppm}$. The cells consisted of Celgard (C480) polyprolylene separator (Celgard Inc., USA), $1 \mathrm{M} \mathrm{LiPF}_{6}$ electrolyte solutions (battery grade, BASF) in ethylene carbonate/ethyl methyl carbonate (EC:DEC) $(1: 1 \mathrm{wt})$, and lithium metal as the counter electrode. An Arbin battery cycler was used to galvanostatically cycle the cells between 3.5 and 4.8 V. X-ray photoelectron spectroscopy (XPS) was performed using a Kratos AXIS Supra with Al K $\alpha$ anode source operated at $15 \mathrm{kV}$. The chamber pressure was $<10^{-8}$ Torr during all measurements and spectra were calibrated using the hydrocarbon $\mathrm{C} 1 \mathrm{~s}$ peak at $284.8 \mathrm{eV}$. Samples were transferred from glove box to XPS chamber via an air-free vacuum transfer system.

Transmission electron microscopy (TEM).-Electrontransparent cross-sectional lamellae were prepared using a FEI Helios NanoLab Dualbeam. The maximum ion beam current used for a regular cross sections is $\sim 3 \mathrm{nA}$ while the pixel dwell time was limited to $100 \mathrm{~ns}$. The samples were extracted out of the thin film following standard lift out procedures and thinned down to $\sim 80 \mathrm{~nm}$ using $0.3 \mathrm{nA}$ cleaning cross sections. STEM-EELS images and spectrums were collected on a JEOL 2100F at $200 \mathrm{kV}$, located at the Center for Functional Nanomaterials at Brookhaven National Laboratory. For all spectra, the beam density measured by the fluorescent screen was $2.4 \mathrm{pA} \mathrm{cm}^{-2}$ and the beam diameter was focused to approximately $0.2 \mathrm{~nm}$. The energy resolution of the electron energy loss spectra was approximately $1 \mathrm{eV}$. For high-loss spectra, a $20 \mathrm{~s}$ pixel dwell time, and $0.2 \mathrm{eV}$ per channel dispersion was used. Selected area electron diffraction (SAED) was collected with the smallest objective aperture $(\sim 150 \mathrm{~nm}$ in diameter). For the deposition temperature dependent study transmission electron microscopy (TEM) bright field and diffraction data were acquired using an FEI Tecnai G2 Sphera TEM equipped with a LaB6 source operating at $200 \mathrm{keV}$. Difftools, a Digital Micrography add-on made by Dave Mitchell, was used to calculate the integrated radial intensity pattern. A power law curve was used to subtract the background.

\section{Results and Discussion}

LLTO target characteristics.-XRD of the sintered ceramic pellet confirms that the target is highly crystalline and consists of the cubic perovskite phase mixed with the tetragonal phase (Fig. 1a). A two phase fit of the cubic phase (space group $P m \overline{3} m$ ) and the tetragonal phase (space group $P 4 / \mathrm{mmm}$ ) was performed resulting in a conventional Rietveld factor $\left(R_{w p}\right)$ of 7.99 . Room temperature EIS measurement using Au blocking electrodes reveals one high frequency semi-circle, one low frequency semi-circle, and a capacitive tail (Fig. 1b). This is in agreement with previous reports designating the high frequency intercept as the lattice conductivity $\left(\mathrm{R}_{\mathrm{L}}\right)$ and the low frequency intercept as the grain boundary conductivity $\left(\mathrm{R}_{\mathrm{GB}}\right)$. The $\mathrm{R}_{\mathrm{L}}$ and $\mathrm{R}_{\mathrm{GB}}$ values were determined using the equivalent circuit displayed in the inset and the respective ionic conductivities were determined using

$$
\sigma=\frac{d}{A R}
$$

where $\mathrm{d}$ is the thickness of the sample, $\mathrm{A}$ is sample area, and $\mathrm{R}$ is the resistance. The pellet has a lattice conductivity of $8.0 \times 10^{-4} \mathrm{~S} / \mathrm{cm}$ and grain boundary conductivity of $2.5 \times 10^{-5} \mathrm{~S} / \mathrm{cm}$, which is consistent with reported values. ${ }^{10,26}$

Deposition pressure dependence.-There is discrepancy between previous reports of optimal amorphous LLTO PLD deposition conditions. Furusawa et al. deposited films in vacuum $\left(5 \times 10^{-6}\right.$ Torr $)$, while Ahn and Yoon deposited at 0.1 Torr $\mathrm{O}_{2}$ partial pressure (Table I). ${ }^{15,19}$ Interestingly, Furusawa et al. produced films with higher ionic conductivity $\left(8.75 \times 10^{-4} \mathrm{~S} / \mathrm{cm}\right)$, but also significantly higher electronic conductivity $\left(4.0 \times 10^{-5} \mathrm{~S} / \mathrm{cm}\right)$. We deposited Pt/aLLTO/Pt vertical films at $400^{\circ} \mathrm{C}, 4 \mathrm{~Hz}$, and $\sim 2 \mathrm{~J} / \mathrm{cm}^{2}$ at various pressures: vacuum $\left(\sim 1 \times 10^{-5}\right.$ Torr $), 0.03$ Torr, and 0.2 Torr $\mathrm{O}_{2}$ partial pressure. DC polarization tests confirm that with higher oxygen pressure the electronic conductivity decreases (Fig. 2a). In fact, for the vacuum and 0.03 Torr sample there is negligible polarization due to the high electronic conductivity. The vacuum film is black, also noted by Furusawa et al., while the 0.03 and 0.2 Torr $\mathrm{O}_{2}$ films are transparent. ${ }^{15}$ All samples are dense films with no pinholes, although 

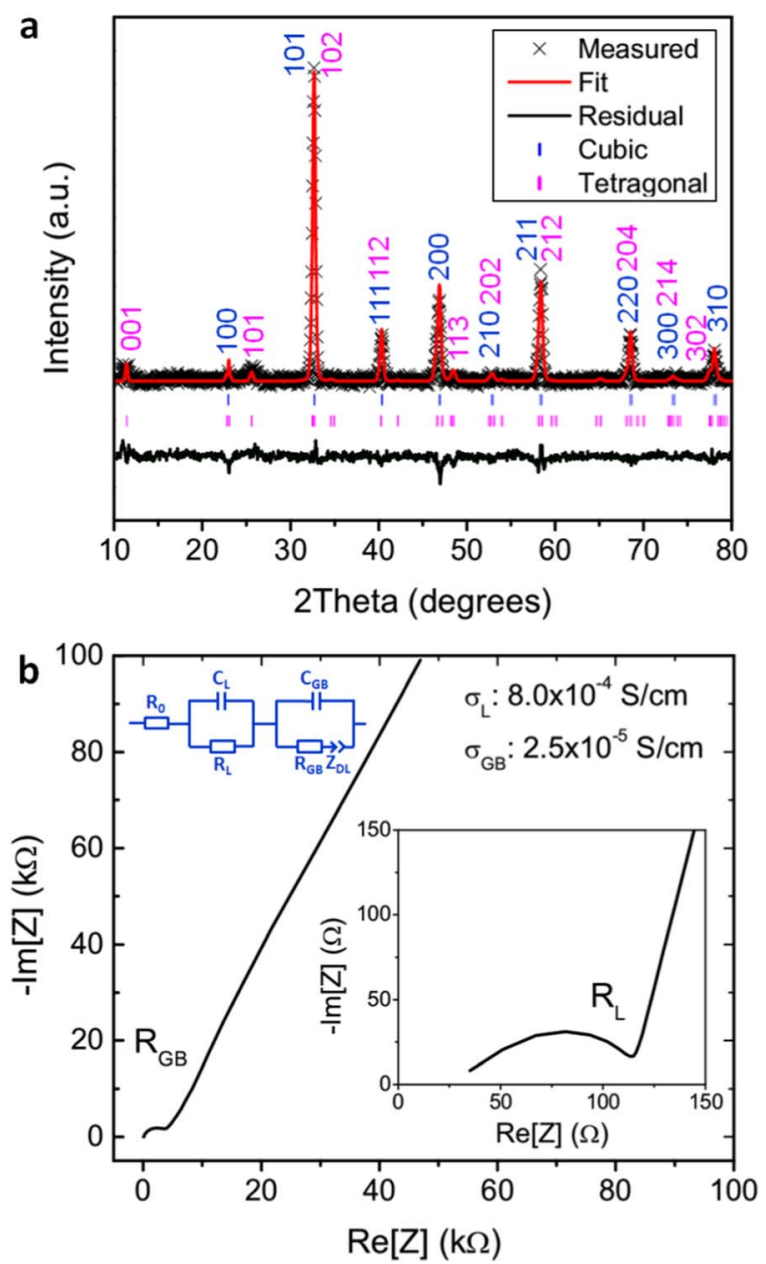

Figure 1. (a) XRD, Rietveld refinement, and (b) EIS of crystalline LLTO pellet.

\section{Table I. Summary of previous reports of amorphous LLTO films} grown by PLD.

\begin{tabular}{lll} 
& Furusawa et al. ${ }^{15}$ & Ahn and Yoon ${ }^{19}$ \\
\hline Pressure & $5 \times 10^{-6}$ Torr & 0.1 Torr \\
Temperature & $25^{\circ} \mathrm{C}$ & $100-600^{\circ} \mathrm{C}$ \\
Frequency & $10 \mathrm{~Hz}$ & $4 \mathrm{~Hz}$ \\
Energy & $180 \mathrm{~mJ} /$ pulse & $2 \mathrm{~J} / \mathrm{cm}^{2}$ \\
Ionic Conductivity & $8.8 \times 10^{-4} \mathrm{~S} / \mathrm{cm}$ & $2.0 \times 10^{-5} \mathrm{~S} / \mathrm{cm}$ \\
Electronic Conductivity & $4.0 \times 10^{-5} \mathrm{~S} / \mathrm{cm}$ & $3.5 \times 10^{-11} \mathrm{~S} / \mathrm{cm}$
\end{tabular}

there appears to be some vertical texturing in the 0.2 Torr sample (Figs. 2b-2d).

STEM-EELS analysis was performed to probe the local bonding structure. Selected area electron diffraction (SAED) shows that while all three samples are amorphous, there are variations in the radial distance of the diffuse rings, indicating shifts in average bond length (Figs. 2e-2g). Plotting the radial intensity we see that there is a $\sim 1.1 \mathrm{~nm}^{-1}$ peak shift between the vacuum sample and 0.2 Torr $\mathrm{O}_{2}$ sample. Also of note, the 0.03 Torr $\mathrm{O}_{2}$ sample has two diffuse rings aligning with both the vacuum and 0.2 Torr $\mathrm{O}_{2}$ peak (Fig. 2h). In addition, EELS analysis was performed and the Ti- $\mathrm{L}_{2,3}$ edge reveals that for the vacuum sample there is a $\sim 0.5 \mathrm{eV}$ chemical shift and intensity reduction in the Ti- $\mathrm{L}_{2}$ edge (Fig. 2i). Gao et al. discovered a similar phenomenon in Ti- $\mathrm{L}_{2,3}$ edge when comparing the La-poor and La-rich regions of crystalline LLTO and attributed the phenomenon to $\mathrm{Ti}^{4+}$ cations reducing to $\mathrm{Ti}^{3+}$ creating oxygen vacancies. ${ }^{11}$ It is reasonable to believe that for LLTO deposited in lower pressure, there is greater oxygen loss resulting in oxygen vacancies. These oxygendeficient domains could result in regions of larger lattice spacing from repulsion of charged atoms, and this excess $\mathrm{Ti}^{3+}$ would also create electron conduction pathways increasing the electronic conductivity. Thus, high oxygen pressure is necessary during pulsed laser deposition to minimize oxygen vacancy formation reducing the electronic conductivity.

Deposition temperature dependence.-There is also inconsistency in previous reports on the optimal deposition temperature (Table I). Crystalline LLTO thin films are deposited at $800^{\circ} \mathrm{C}$ and
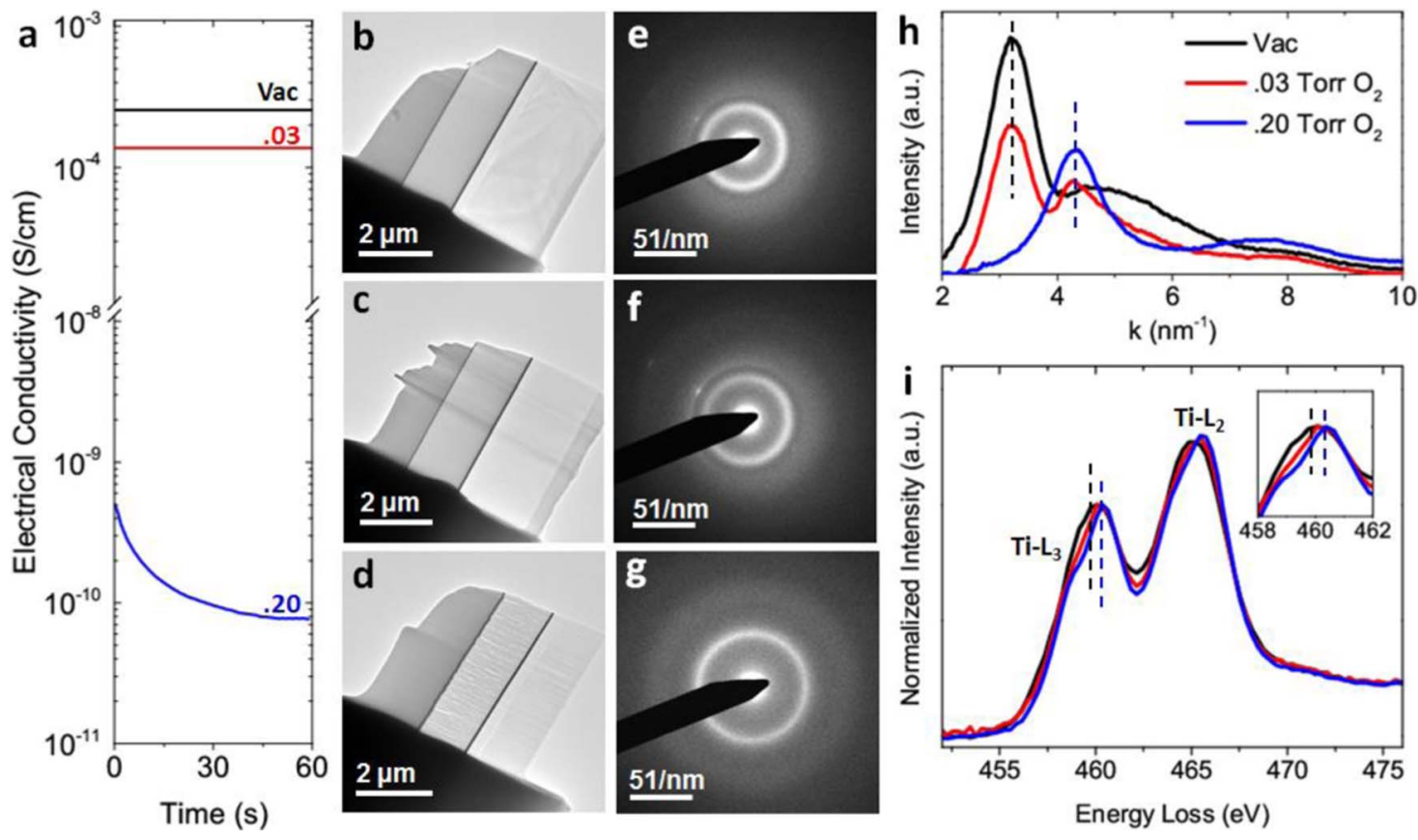

Figure 2. (a) DC conductivity of a-LLTO thin films at room temperature. TEM bright field image and SAED of samples prepared in (b, e) vacuum, (c, f) .03 Torr $\mathrm{O}_{2}$, and (d, g) .2 Torr $\mathrm{O}_{2}$ chamber pressure. Corresponding (h) intensity profile and (i) normalized Ti-L edge spectra. 


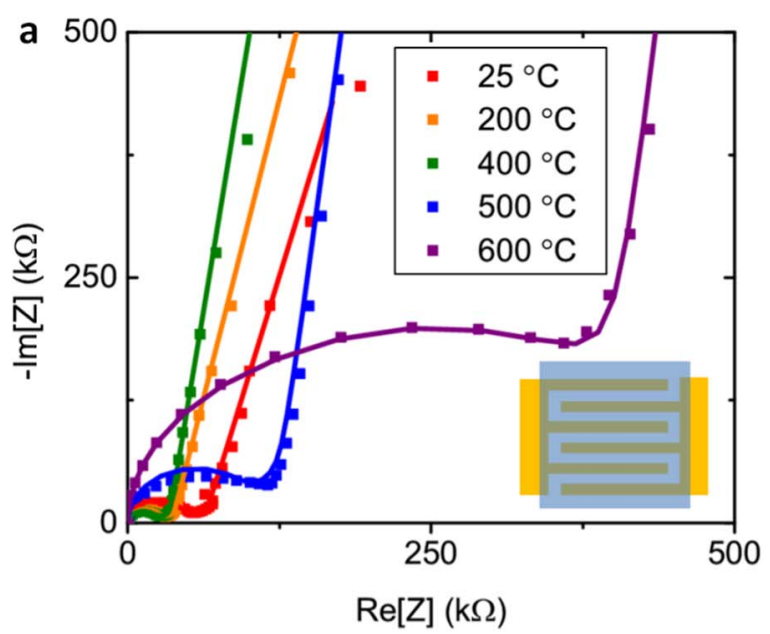

b
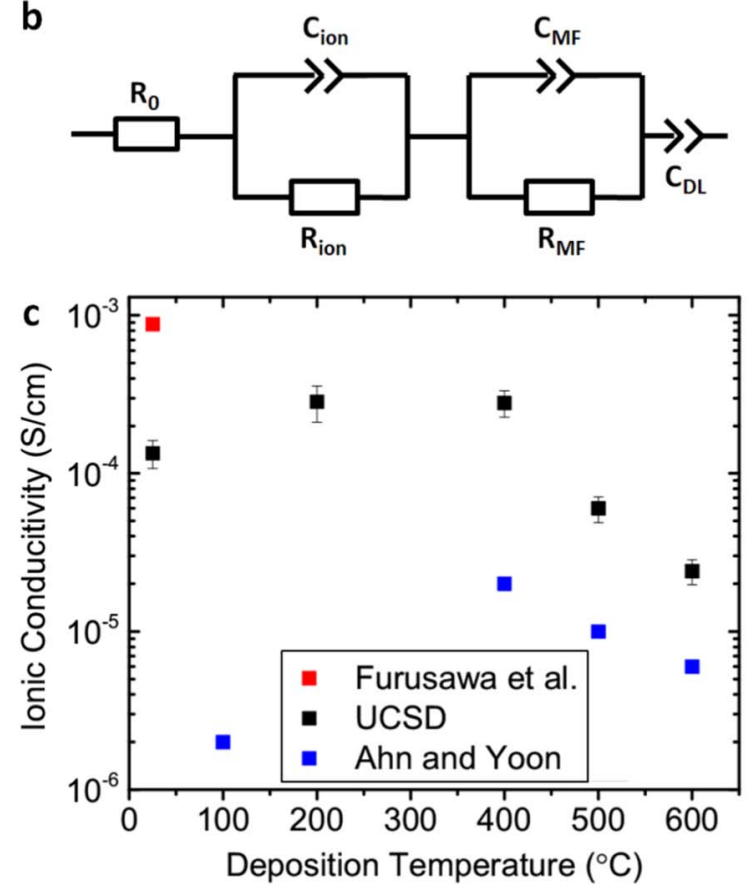

Figure 3. (a) Nyquist plot of a-LLTO thin film samples deposited at various temperatures with interdigitated contacts. (b) Equivalent circuit corresponding to Nyquist plot. (c) Variation in ionic conductivity as a function of deposition temperature compared to previous literature..$^{15,19}$

LLTO will remain amorphous as long as the deposition temperature is $<700^{\circ} \mathrm{C}^{28}$ We deposited a-LLTO on interdigitated contacts at 0.2 Torr oxygen, $4 \mathrm{~Hz}$, and $\sim 2 \mathrm{~J} / \mathrm{cm}^{2}$ at various temperatures expanding the full range from Furusawa et al. and Ahn and Yoon. ${ }^{15,19}$ The Nyquist plots show a single semicircle and dielectric capacitance tail (Fig. 3a). The data was fit using the equivalent circuit in Fig. 3b, which is consistent with the models used for lithium phosphorous oxynitride (LiPON) thin film electrolytes. ${ }^{29}$ Since the films are amorphous, there are no separate lattice and grain boundary parameters, but two RC circuits are needed to fit the data. This is likely due to sample roughness and/or contact interfacial phenomenon. Plotting the ionic conductivity across temperature we see that our films are on par with previous literature (Fig. 2c). Films deposited at $200^{\circ} \mathrm{C}$ and $400^{\circ} \mathrm{C}$ showed the highest ionic conductivity of $3.0 \times 10^{-4} \mathrm{~S} / \mathrm{cm}$. Additionally, at high temperature our films show a similar trend to Ahn and Yoon, where the samples decline in ionic conductivity above $400^{\circ} \mathrm{C} .{ }^{19}$
Grazing angle XRD indicates that films deposited up to $600^{\circ} \mathrm{C}$ remain amorphous, with only peaks from the platinum coated substrate (Fig. S1a). However, SAED of a-LLTO deposited at $600^{\circ} \mathrm{C}$ features both an amorphous diffuse ring and diffuse diffraction spots (Fig. $\mathrm{S} 1 \mathrm{c}$.). The sample is still overwhelmingly amorphous, and these peaks cannot be indexed to a particular crystal structure, but it is likely that at higher temperatures LLTO nanocrystals begin to form. Any crystallization will be detrimental to ionic conductivity due to grain boundary diffusion. Crystalline LLTO thin films deposited by PLD have been shown to be up to an order of magnitude lower in ionic conductivity than amorphous. ${ }^{15}$ Thus, for good ionic conductivity we must keep the deposition temperature $\leq 400^{\circ} \mathrm{C}$.

The temperature series conditions were also deposited in the $\mathrm{Pt} / \mathrm{LLTO} / \mathrm{Pt}$ vertical configuration to confirm its compatibility with integration into a thin film device. Given the geometrical constraints and high ionic conductivity the semicircle was too small to detect at room temperature. Instead there is only the capacitive tail. To obtain an accurate calculation of ionic conductivity the samples were cooled to various temperatures. At lower temperatures the ionic conductivity decreases, increasing the resistance and signal (Fig. 4a). From the Arrhenius plot we are able to extrapolate activation energy on par with previous experimental and computational results and a room temperature ionic conductivity, which was in agreement with the interdigitated contact values (Fig. 4b). ${ }^{15-17,30}$ The electronic and ionic conductivity is summarized in Fig. 5. Similar to Furusawa et al. room temperature PLD resulted in a thin film with high ionic conductivity, but also high electronic conductivity. ${ }^{15}$ The electronic conductivity decreased with higher temperature, probably correlating with greater oxygen incorporation kinetics. For a good solid-state electrolyte there needs to be several orders of magnitudes between the high ionic and low electronic conductivity. Therefore, the optimal deposition temperature should be at $400^{\circ} \mathrm{C}$.

Electrochemistry.-The optimized a-LLTO deposition conditions $\left(0.2\right.$ Torr $\left.\mathrm{O}_{2}, 400^{\circ} \mathrm{C}, \sim 2 \mathrm{~J} / \mathrm{cm}^{2}, 4 \mathrm{~Hz}\right)$ were used to coat a $\mathrm{LiNi}_{0.5} \mathrm{Mn}_{1.5} \mathrm{O}_{4}$ (LNMO) thin-film electrode, thus referred to as the LNMO/a-LLTO electrode. The cycling performance of the LNMO and LNMO/a-LLTO electrode are in Fig. 6. The a-LLTO deposition does not alter the LNMO intercalation chemistry as both cells show the characteristic voltage profile for LNMO, exhibiting the $\mathrm{Ni}^{2+} / \mathrm{Ni}^{4+}$ $(4.7 \mathrm{~V})$ and $\mathrm{Mn}^{3+} / \mathrm{Mn}^{4+}(4.0 \mathrm{~V})$ redox couples. For a pure phase LNMO film, we shouldn't observe this $4 \mathrm{~V} \mathrm{Mn}^{3+} / \mathrm{Mn}^{4+}$ redox signal, but $\mathrm{Mn}^{3+}$ ions have been previously found in composite and PLD electrodes. ${ }^{31,32}$ This is potentially due to non-stoichiometric oxygen or nickel transfer during PLD. The LNMO/a-LLTO cell exhibits superior reversible capacity stability with $98 \%$ discharge capacity retention after 50 cycles (Fig. 6b). This corresponds to a $0.036 \%$ capacity fade per cycle. However, the coulombic efficiency is relatively low for both cells at $96 \%$. This charge loss is due to electrochemical decomposition of the liquid electrolyte at extremely high voltage such as $4.8 \mathrm{~V}$ during each cycle. And going to a full solid-state device would overcome such effects.

For the LNMO/a-LLTO electrode there is no significant change in the voltage profile and the cell maintains comparable discharge capacity for a variety of cycling rates (Fig. S2). The interfacial compatibility between the LNMO and a-LLTO is crucial for cell performance and previous attempts to pair PLD a-LLTO with $\mathrm{LiCoO}_{2}$ (LCO) resulted in extreme performance deterioration from a highly resistive interfacial layer. ${ }^{19}$ The excellent capacity retention is indicative of minimal formation of an unfavorable interfacial LNMO/a-LLTO reaction, and this is further investigated with EIS (Fig. S3). XPS analysis confirms that the a-LLTO remains on the LNMO electrode surface with no dissolution during cycling (Fig. S4). Thus, we have shown that a-LLTO has good rate performance and is electrochemically compatible with LNMO for future high voltage thin film battery devices. 
a

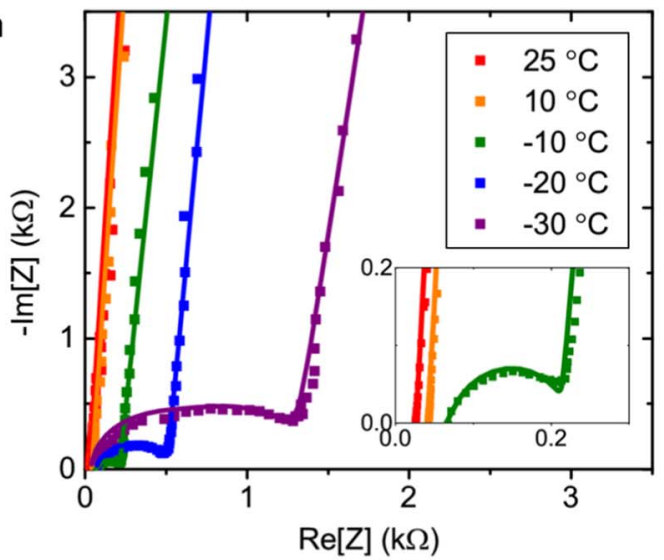

b

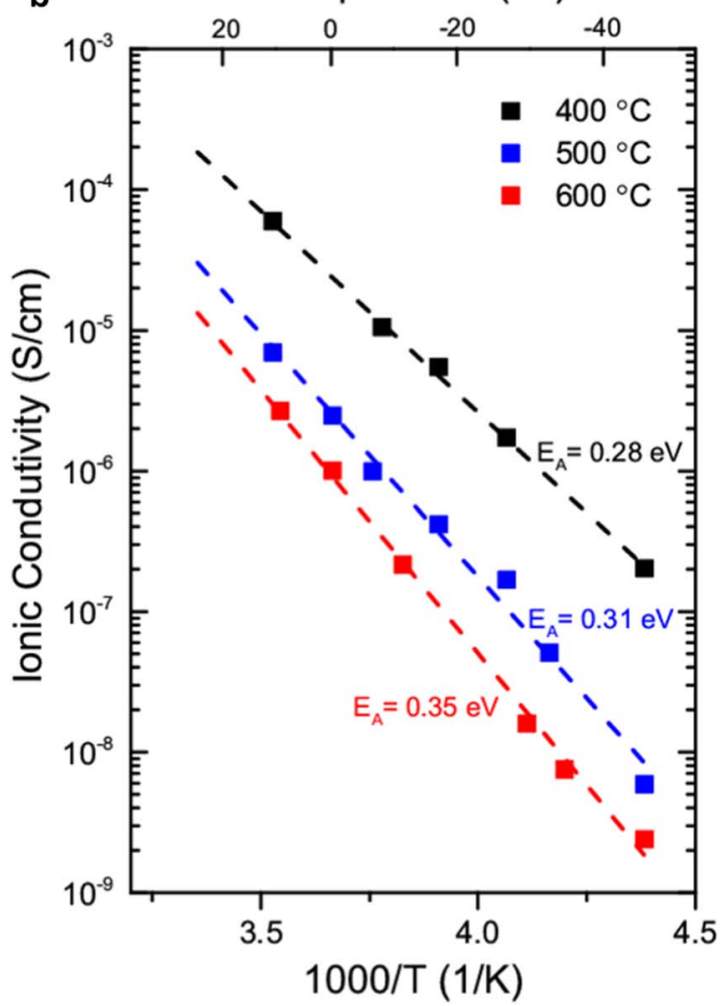

Figure 4. (a) Nyquist plot at various temperatures of a-LLTO thin film sample deposited at $400^{\circ} \mathrm{C}$ with vertical contacts. (b) Arrhenius plot of various deposition temperatures.

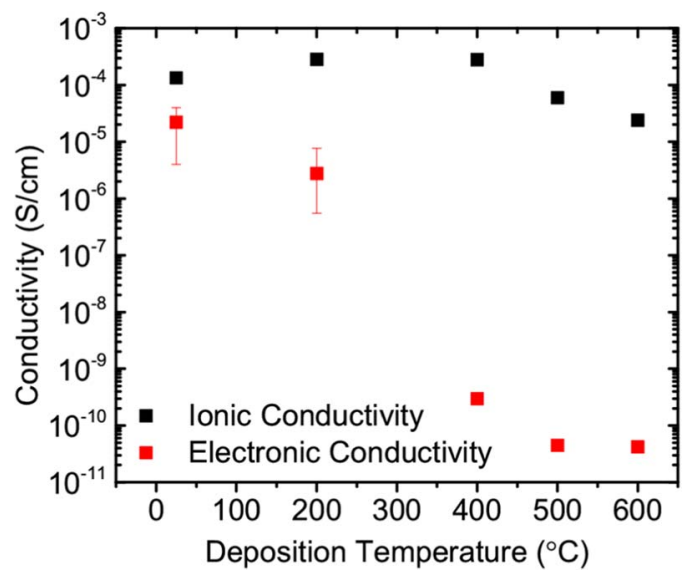

Figure 5. Ionic and electronic conductivity at various temperatures.
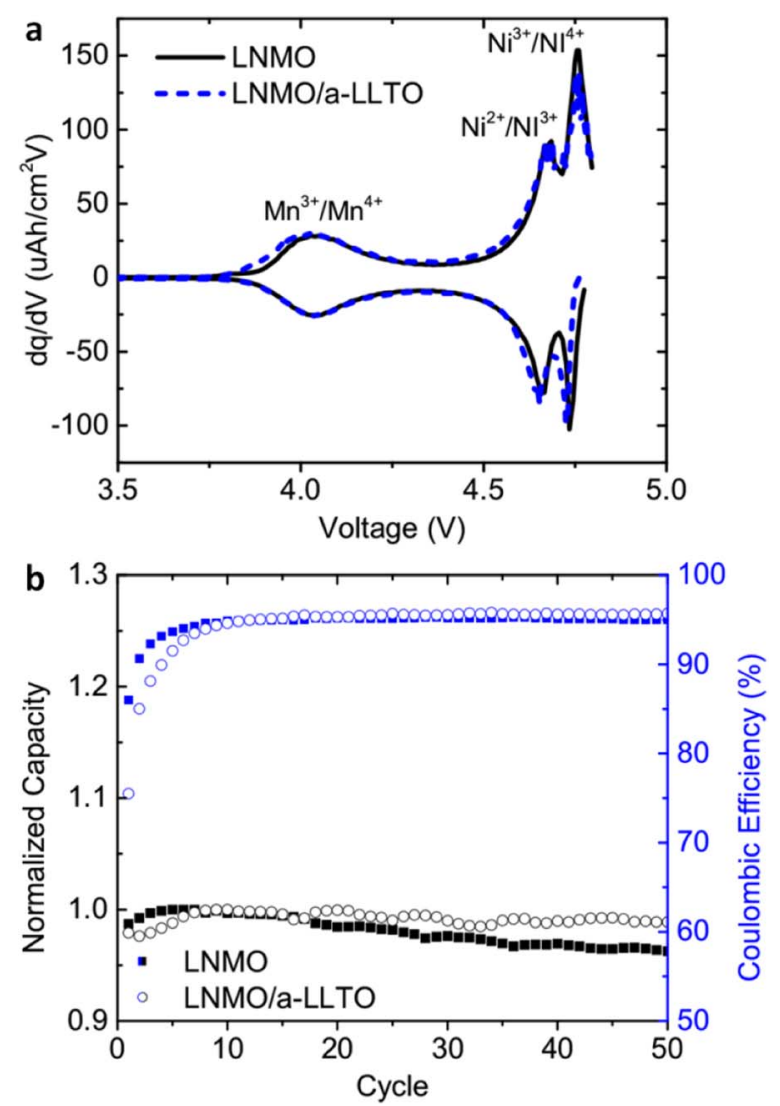

Figure 6. (a) Cycling profile and (b) performance of $300 \mathrm{~nm} \mathrm{LiNi}_{0.5} \mathrm{Mn}_{1.5} \mathrm{O}_{4}$ and $300 \mathrm{~nm} \mathrm{LiNi} 0.5 \mathrm{Mn}_{1.5} \mathrm{O}_{4}$ with $500 \mathrm{~nm}$ a-LLTO coating.

\section{Conclusions}

In this work, we prepared amorphous LLTO thin films by pulsed laser deposition for use in high voltage thin film lithium-ion batteries. Various deposition conditions were optimized to maximize ionic conductivity while maintaining sufficiently low electronic conductivity to function as an effective solid-state electrolyte. Our $\sim 1.2$ $\mu \mathrm{m}$ thick a-LLTO film grown at 0.2 Torr $\mathrm{O}_{2}, 400^{\circ} \mathrm{C}, \sim 2 \mathrm{~J} / \mathrm{cm}^{2}$, and $4 \mathrm{~Hz}$ exhibits an ionic conductivity of $3.0 \times 10^{-4} \mathrm{~S} / \mathrm{cm}$ and electronic conductivity of $3.0 \times 10^{-10} \mathrm{~S} / \mathrm{cm}$. This condition is used to fabricate a LNMO/a-LLTO half-cell, which maintained $98 \%$ capacity retention after 50 cycles. There was no significant degradation in cycling performance indicative of excellent compatibility between LNMO electrode and a-LLTO electrolyte. Future work will be done to further examine the LNMO/a-LLTO interfacial stability and develop a high voltage LNMO/a-LLTO based thin film solid-state battery.

\section{Acknowledgments}

The authors thank Cyrus S. Rustomji for fruitful discussion and use of environmental chamber. This work is supported by the U.S. Department of Energy, Office of Basic Energy Sciences, under Award Number DE-SC0002357. This research used resources of the Center for Functional Nanomaterials, which is a U. S. DOE Office of Science Facility, at Brookhaven National Laboratory under Contract No. DE-SC0012704. This work was performed in part at the San Diego Nanotechnology Infrastructure (SDNI), a member of the National Nanotechnology Coordinated Infrastructure, which is supported by the National Science Foundation (grant ECCS-1542148). XRD and XPS were performed at the UC Irvine Materials Research Institute (IMRI) using instrumentation funded in part by the National Science Foundation Major Research Instrumentation Program under grant no. CHE-1338173. We acknowledge the use of the UCSD Cryo-Electron 
Microscopy Facility which is supported by NIH grants to Dr. Timothy S. Baker and a gift from the Agouron Institute to UCSD. J. L. acknowledges support from the Eugene Cota-Robles Fellowship Program of the University of California San Diego.

\section{References}

1. A. C. Luntz, J. Voss, and K. Reuter, J Phys Chem Lett, 6 (22), 4599 (2015).

2. V. P. Phan, B. Pecquenard, and F. Le Cras, Adv Funct Mater, 22 (12), 2580 (2012).

3. J. Li, C. Ma, M. Chi, C. Liang, and N. J. Dudney, Advanced Energy Materials, 1401408 (2014).

4. F. Xu, N. J. Dudney, G. M. Veith, Y. Kim, C. Erdonmez, W. Lai, and Y. M. Chiang, Journal of Materials Research, 25 (8), 1507 (2010).

5. N. Kamaya, K. Homma, Y. Yamakawa, M. Hirayama, R. Kanno, M. Yonemura, T. Kamiyama, Y. Kato, S. Hama, K. Kawamoto, and A. Mitsui, Nat Mater, 10 (9), $682(2011)$.

6. C. Cao, Z.-B. Li, X.-L. Wang, X.-B. Zhao, and W.-Q. Han, Frontiers in Energy Research, 2 (2014)

7. O. Bohnke, Solid State Ionics, 179 (1-6), 9 (2008).

8. Z. F. Zheng, H. Z. Fang, Z. K. Liu, and Y. Wang, J Electrochem Soc, 162 (1), A244 (2015).

9. A. G. Belous, G. N. Novitskaya, S. V. Polyanetskaya, and Y. I. Gornikov, Inorg Mater, 23 (3), 412 (1987).

10. Y. Inaguma, L. Q. Chen, M. Itoh, T. Nakamura, T. Uchida, H. Ikuta, and M. Wakihara, Solid State Commun, 86 (10), 689 (1993).

11. X. Gao, C. A. J. Fisher, T. Kimura, Y. H. Ikuhara, H. Moriwake, A. Kuwabara, H. Oki, T. Tojigamori, R. Huang, and Y. Ikuhara, Chemistry of Materials, 25 (9), 1607 (2013).

12. S. Stramare, V. Thangadurai, and W. Weppner, Chemistry of Materials, 15 (21), 3974 (2003).

13. K. Y. Yang, I. C. Leu, K. Z. Fung, M. H. Hon, M. C. Hsu, Y. J. Hsiao, and M. C. Wang, Journal of Materials Research, 23 (7), 1813 (2007).
14. C. H. Chen and K. Amine, Solid State Ionics, 144 (1-2), 51 (2001).

15. S. Furusawa, H. Tabuchi, T. Sugiyama, S. W. Tao, and J. T. S. Irvine, Solid State Ionics, 176 (5-6), 553 (2005).

16. Z. F. Zheng, H. Z. Fang, F. Yang, Z. K. Liu, and Y. Wang, J Electrochem Soc, 161 (4), A473 (2014).

17. Y. L. Xiong, H. Z. Tao, J. A. Zhao, H. Cheng, and X. J. Zhao, J Alloy Compd, 509 (5), 1910 (2011)

18. C. L. Li, B. Zhang, and Z. W. Fu, Thin Solid Films, 515 (4), 1886 (2006).

19. J. K. Ahn and S. G. Yoon, Electrochem Solid St, $8(2)$, A75 (2005).

20. J. K. Ahn and S. G. Yoon, Electrochim Acta, 50 (2-3), 371 (2004).

21. M. Morales, P. Laffez, D. Chateigner, and I. Vickridge, Thin Solid Films, 418 (2), 119 (2002).

22. T. Aaltonen, M. Alnes, O. Nilsen, L. Costelle, and H. Fjellvag, J Mater Chem, 20 (14), 2877 (2010)

23. K. Kitaoka, H. Kozuka, T. Hashimoto, and T. Yoko, J Mater Sci, 32 (8), 2063 (1997)

24. E. J. van den Ham, N. Peys, C. De Dobbelaere, J. D'Haen, F. Mattelaer, C. Detavernier, P. H. L. Notten, A. Hardy, and M. K. Van Bael, J Sol-Gel Sci Techn, 73 (3), $536(2015)$

25. A. Manthiram, K. Chemelewski, and E. S. Lee, Energy \& Environmental Science, 7 (4), 1339 (2014)

26. A. C. Sutorik, M. D. Green, C. Cooper, J. Wolfenstine, and G. Gilde, J Mater Sci, 47 (19), 6992 (2012).

27. H. Xia, S. B. Tang, L. Lu, Y. S. Meng, and G. Ceder, Electrochim Acta, 52 (8), 2822 (2007).

28. H. T. K. Kamala Bharathi, S. Takeuchi, L. Meshi, H. Shen, J. Shin, I. Takeuchib, and L. A. Bendersky, RSC Advances, 6, 9 (2016).

29. J. C. Li, N. J. Dudney, J. Nanda, and C. D. Liang, Acs Appl Mater Inter, 6 (13), 10083 (2014).

30. D. N. Qian, B. Xu, H. M. Cho, T. Hatsukade, K. J. Carroll, and Y. S. Meng, Chemistry of Materials, 24 (14), 2744 (2012).

31. Q. M. Zhong, A. Bonakdarpour, M. J. Zhang, Y. Gao, and J. R. Dahn, J Electrochem Soc, 144 (1), 205 (1997).

32. H. Xia and L. Lu, Phys Scripta, T129, 43 (2007). 